\title{
Videofluoroscopic and Manometric Evaluation of Pharyngeal and Upper Esophageal Sphincter Function During Swallowing
}

\author{
Kyung Jae Yoon, ${ }^{1}$ Jung Ho Park, ${ }^{2 *}$ Jung Hwan Park ${ }^{3}$ and II Seok Jung ${ }^{3}$ \\ Departments of ${ }^{1}$ Physical and Rehabilitation, and ${ }^{2}$ Internal Medicine, Kangbuk Samsung Hospital, Sungkyunkwan University College of \\ Medicine, Seoul, Korea; and ${ }^{3}$ Department of Bionano Technology, Gachon University, Seongnam, Gyeonggi-do, Korea
}

\begin{abstract}
Background/Aims
The purpose of this study was to determine important manometric metrics for the analysis of pharyngeal and upper esophageal sphincter (UES) function and to investigate the effect of viscosity and other confounding factors on manometric results.

\section{Methods}

Manometric studies were performed on 26 asymptomatic volunteers (12 men and 14 women; age, 19-81 years). The manometric protocol included 5 water swallows $(5 \mathrm{~mL}), 5$ barium swallows $(5 \mathrm{~mL})$ and 5 yogurt swallows $(5 \mathrm{~mL})$. Evaluation of high-resolution manometry parameters including basal pressure of the UES, mesopharyngeal contractile integral (mesopharyngeal $\mathrm{Cl}, \mathrm{mmHg} \cdot \mathrm{cm} \cdot \mathrm{sec}$ ), $\mathrm{Cl}$ of the hypopharynx and UES (hypopharyngeal $\mathrm{Cl}$ ), relaxation interval of UES, median intrabolus pressure and nadir pressure at UES was performed using MATLAB.
\end{abstract}

\section{Results}

Mesopharyngeal Cls for barium and yogurt swallows were significantly lower than those for water swallows (both $P<0.05)$. Hypopharyngeal Cls for water swallows were significantly lower than those for barium swallows $(P=0.004)$, and median bolus pressure at UES for barium swallows was significantly higher than that for water and yogurt swallows (both $P<0.05)$. Furthermore, hypopharyngeal $\mathrm{Cl}$ and median intrabolus pressure at UES were significantly related to age for 3 swallows (all $P$ $<0.01$ and $P<0.05$, respectively). A significant negative correlation was also noted between nadir pressure at UES and age for water and yogurt swallows (all $P<0.05$ ).

\section{Conclusions}

Manometric measurement of the pharynx and UES varies with respect to viscosity. Moreover, age could be a confounding variable in the interpretation of pharyngeal manometry.

(J Neurogastroenterol Motil 2014;20:352-361)

Key Words

Manometry; Deglutition; Esophageal sphincter, upper; Fluoroscopy; Pharynx

Received: February 27, 2014 Revised: March 24, 2014 Accepted: April 2, 2014

(c) This is an Open Access article distributed under the terms of the Creative Commons Attribution Non-Commercial License (http://creativecommons. org/licenses/by-nc/3.0) which permits unrestricted non-commercial use, distribution, and reproduction in any medium, provided the original work is properly cited.

*Correspondence: Jung Ho Park, MD

Department of Internal Medicine, Kangbuk Samsung Hospital, 29, Saemunan-ro, Jongno-gu, Seoul 110-746, Korea

Tel: +82-2-2001-2059, Fax: +82-2-2001-2485, E-mail: pjho3@hotmail.com

Financial support: None.

Conflicts of interest: None.

Author contributions: Kyung Jae Yoon, writing a paper; Jung Ho Park, design a study; Jung Hwan Park and II Seok Jung, making a MATLAB program. ORCID: Jung Ho Park, http://orcid.org/0000-0003-2568-128X. 


\section{Introduction}

Swallowing is a complex process involving multiple oropharyngeal and upper esophageal sphincter (UES) muscles. Coordinated contractions of these muscles are under the control of the autonomic nervous system, and even a small damage to neurons can cause swallowing problems. Videofluoroscopic swallow evaluation (VFSE) and fiberoptic endoscopic evaluation of swallowing (FEES) are 2 major tests used for the evaluation of oropharyngeal dysphagia. VFSE can visualize the rapid sequence of swallowing events and helps to identify pathologic movements of the bolus, such as aspiration and pooling in the pyriform sinus and valleculae. ${ }^{1,2}$ However, interpretation of the results from VFSE and FEES is subjective, and can result in poor intraobserver reliability. ${ }^{3,4}$ Moreover, they can only show pathologic findings resulting from neuromuscular abnormalities instead of direct causes of dysphagia, and quantitative functional evaluation of pharyngeal and UES muscles is impossible with these methods.

Accurate manometric evaluation of swallowing can be complicated by the temporal contractile rate of swallowing related muscles ${ }^{6}$ and upward movement of the UES during swallowing. ${ }^{7}$ However, these problems have been solved by the introduction of high-resolution manometry (HRM). The rapid contractile response can be accurately recorded with $\mathrm{HRM},{ }^{8}$ and 36 solid state sensors spaced at $1-\mathrm{cm}$ intervals can overcome the problems related to UES movement during deglutition. ${ }^{9}$ Several studies already have reported detailed analyses of UES and pharyngeal muscles with a HRM and suggested normative values for manometric data. ${ }^{5,10-12}$

However, there are several points to consider in previous manometric analyses of UES and pharyngeal function. First, little data is available concerning the correlation between manometric and VFSE findings. HRM and VFSE could be complementary to each other. Therefore, it would be more helpful to have the 2 tests performed simultaneously for a better understanding of the mechanism of dysphagia. Second, previous studies have used peak or mean pharyngeal pressure as a metric for pharyngeal contractility. However, in the analysis of esophageal motility, contractile integral (CI) was suggested to be a more reliable index for contractility. $\mathrm{CI}$ is the volume of contraction when depicted as a 3 dimensional solid with the $\mathrm{x}, \mathrm{y}$, and $\mathrm{z}$-axes representing time, spatial domain and pressure magnitude, respectively. ${ }^{13}$ Third, manometric findings of patients with dysphagia (especially UES relaxation) are usually very complicated, so it is not easy to use complex functions for the analysis of metrics. A simpler definition of UES relaxation interval may be more helpful for the analysis of UES function of patients with dysphagia. Finally, there are several factors that affect manometric measurements, including age, gender ${ }^{14,15}$ and viscosity. ${ }^{5}$

The aims of this study were to determine important manometric metrics for the analysis of pharyngeal and UES function, and to investigate the effect of various confounding factors on manometric results.

\section{Materials and Methods}

\section{Participants}

Twenty-six healthy adults were enrolled as participants via public advertisement or word of mouth. Subjects had no history of swallowing difficulty or upper gastrointestinal tract surgery, and no other significant medical conditions. The study protocol was approved by the Sungkyunkwan University Institutional Review Board, and informed consent was obtained from each participant.

\section{High-resolution Manometry}

A solid-state manometric assembly with 36 circumferential sensors spaced at $1-\mathrm{cm}$ intervals (4.2 $\mathrm{mm}$ outer diameter) was used (Given Imaging, Los Angeles, CA, USA). Before the recording, transducers were calibrated at 0 and $300 \mathrm{mmHg}$ using externally applied pressure.

\section{Study Protocol}

\section{Simultaneous examination of videofluoroscopic swallow evaluation and high-resolution manometry}

After positioning a manometric catheter as described below, VFSE and HRM studies were performed at the same time with subjects seated in a chair. As soon as $5 \mathrm{~mL}$ of diluted barium $(50 \%)$ was administered, a lateral view of swallowing was recorded, fluoroscopy and manometric data were acquired, and then stored using Manoview software (Given Imaging).

\section{Effect of food viscosity on manometric results during pharyngeal phase of swallowing}

After a brief interview and examination to ensure the absence of gastrointestinal symptoms and to perform anthropometric 
measurements, subjects underwent transnasal placement of the manometric assembly. Studies were done in the sitting position after at least an 8-hour fast. The manometric catheter was positioned to record from the hypopharynx to the UES and was fixed in place by taping it to the nose. The manometric protocol included a 5-minute period to assess basal sphincter pressure, 5 swallows of $5 \mathrm{~mL}$ water, 5 swallows of $5 \mathrm{~mL}$ yogurt (1,200 centi poise) and 5 swallows of $5 \mathrm{~mL}$ diluted barium $(50 \%, 43.4$ centi poise).

\section{Manometry Data Analysis}

Manometric data were analyzed using MATLAB (MathWorks; Natick, MA, USA) customized for processing binary manometric data into isocontour pressure plots and spatial pressure variation plots. This was done by exporting the binary manometry data from ManoView in the ASCII text format for processing and storage. These ASCII files were then reconverted into a double-precision binary format for use in MATLAB, and isocontour or spatial pressure variation plots were generated (Fig. 1).

Six important metrics of HRM were chosen for comparison
A

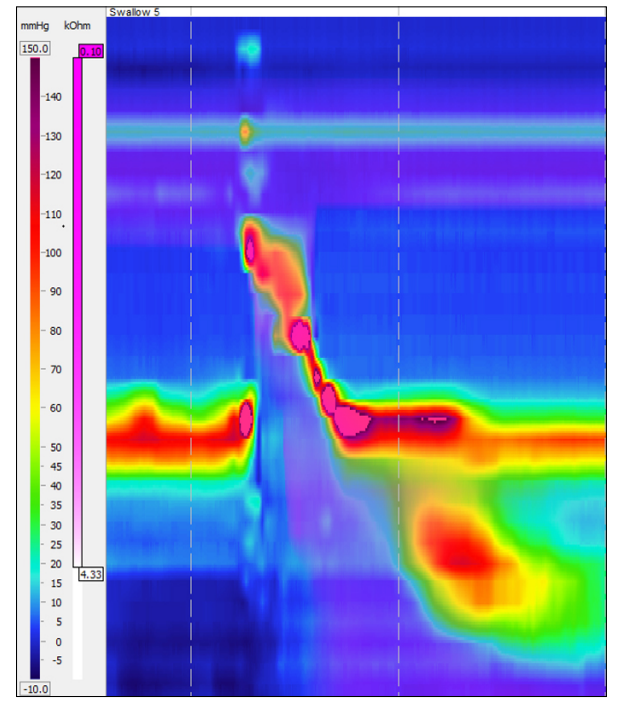

B

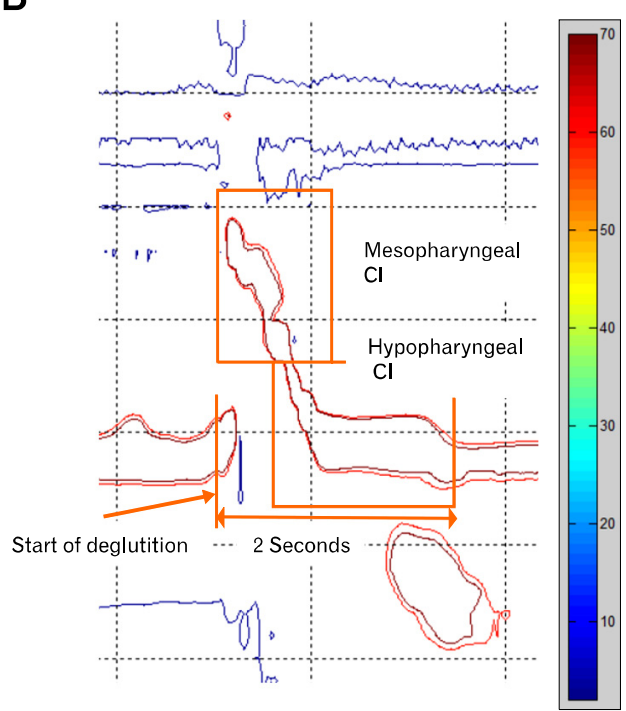

Figure 1. Isobaric contour image generated from binary manometric data. (A) Manoview image of water $5 \mathrm{~mL}$ swallow (B) Isobaric contour generated from MATLAB program. Mesopharyngeal contractile integral (CI) was calculated by amplitude $\mathrm{X}$ duration $\mathrm{X}$ length $(\mathrm{mmHg} \cdot \mathrm{cm} \cdot \mathrm{sec})$ of muscular contraction $\geq 70 \mathrm{mmHg}$. CI of hypopharynx and upper esophageal sphincter (hypopharyngeal CI) was calculated in the same manner by amplitude $\mathrm{X}$ duration $\mathrm{X}$ length $(\mathrm{mmHg} \cdot \mathrm{cm} \cdot \mathrm{sec})$ of muscular contraction $\geq 70 \mathrm{mmHg}$ for 2 seconds after onset of deglutition.
A

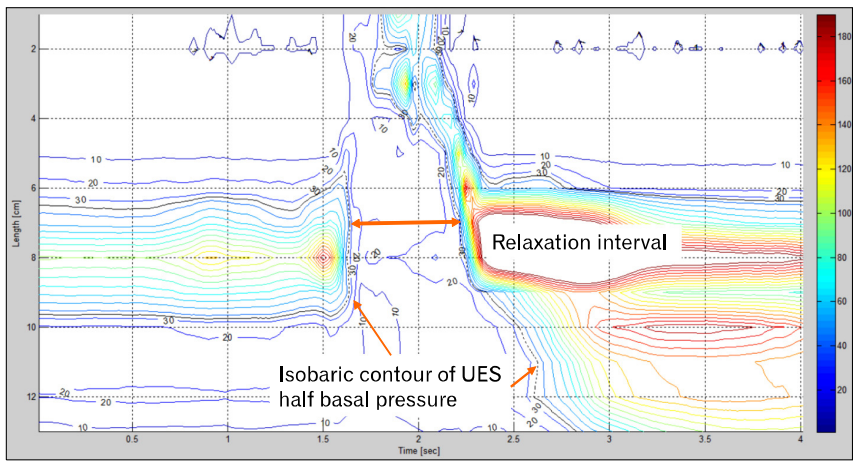

B

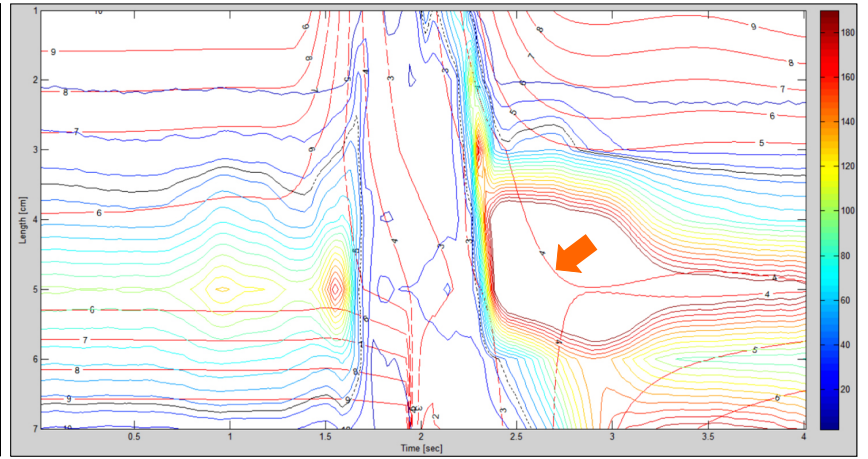

Figure 2. Isobaric and isoimpedance contour image within the upper esophageal sphincter (UES) during deglutitive relaxation. (A) Isobaric contour of the UES during deglutitive relaxation generated from MATLAB. Relaxation interval was defined as the duration of time from the onset at the point of departure from half the baseline to the offset at the return to half baseline pressure (indicated by dotted line) (B) Isobaric and isoimpedance contour during the UES relaxation interval. As the isoimpedance line gets closer to the starting point of UES contraction, the intervals between the lines become narrower and impedance increased. When relaxation comes to an end, low impedance lines change into lines with higher impedance, and the isoimpedance line changes its direction toward the right side of the image, not downward (orange arrow). 
between swallows including: (1) basal pressure of the UES, (2) mesopharyngeal CI $(\mathrm{mmHg} \cdot \mathrm{cm} \cdot \mathrm{sec})$, (3) CI of the hypopharynx and UES (hypopharyngeal CI $[\mathrm{mmHg} \cdot \mathrm{cm} \cdot \mathrm{sec}]$ ), (4) relaxation interval of UES (sec), (5) median intrabolus pressure and (6) nadir pressure at UES (Fig. 1 and 2).

Basal pressure of UES was defined as the maximal pressure occurring during the normal respiratory cycle. To minimize sampling error, the mean of 5 consecutive values was derived (using the same methodology as that for the analysis of resting esophagogastric junction pressure parameters). ${ }^{16}$ Mesopharyngeal CI was calculated by amplitude $\mathrm{X}$ duration $\mathrm{X}$ length $(\mathrm{mmHg} \cdot \mathrm{cm} \cdot \mathrm{sec})$ of muscular contraction $\geq 70 \mathrm{mmHg}$. The reference value; 70 $\mathrm{mmHg}$ for $\mathrm{CI}$ of the mesopharynx was derived from the average pressure $(67.47 \pm 21.22 \mathrm{mmHg})$ generated by the tongue and posterior palate during water swallowing. ${ }^{11}$ Hypopharyngeal CI was calculated in the same manner by amplitude $\mathrm{X}$ duration $\mathrm{X}$ length $(\mathrm{mmHg} \cdot \mathrm{cm} \cdot \mathrm{sec})$ of muscular contraction $\geq 70 \mathrm{mmHg}$ for 2 seconds after the onset of deglutition. In this study, the median value of the basal UES was $73.6 \mathrm{mmHg}$ and so $70 \mathrm{mmHg}$ was determined as a reference value for hypopharyngeal CI.

Relaxation interval was defined as the duration of time from onset at the point of departure from half the baseline to the offset at the return to half baseline pressure. ${ }^{8}$ Median intrabolus pressure was the median pressure recorded during the relaxation interval. Nadir pressure was minimum UES pressure attained during relaxation. ${ }^{17}$

\section{Statistical Methods}

All data are expressed as mean values \pm standard deviation or median values (interquartile range; IQR). Comparisons of metrics among 3 swallow types were made by the paired $t$ test and ANOVA test. The correlation between manometric parameters and confounding factors (age, sex, height, body mass index $[\mathrm{BMI}]$, and waist and hip circumferences) was computed with Pearson's correlation. The alpha level of significance was set at $P$ $<0.05$. All analyses were performed using SPSS version 18 (SPSS, Chicago, IL, USA).

\section{Results}

Twenty-six participants ( 12 males and 14 females) completed the manometric protocol. The mean age was $42.5 \pm 18.7$ years (19-29 years, $31 \%$; 30-39 years, $31 \%$; $40-49$ years, $12 \%$ and 50-81 years, 26\%). The mean height and weight were $164.9 \pm$ $8.6 \mathrm{~cm}$ and $61.2 \pm 10.0 \mathrm{~kg}$, respectively. The mean BMI, waist circumference, and hip circumference was $22.4 \pm 23 \mathrm{~kg} / \mathrm{m}^{2}, 79.3$ $\pm 10.3 \mathrm{~cm}$, and $95.3 \pm 6.2 \mathrm{~cm}$, respectively. Two out of $26 \mathrm{pa}-$ tients $(8 \%)$ were overweight $\left(\mathrm{BMI}>25 \mathrm{~kg} / \mathrm{m}^{2}\right)$.

\section{Simultaneous Examination of Videofluoroscopic Swallow Evaluation and High-resolution Manometry}

The examinations were repeated 3 times for each patient. Pictures of VFSE were compared and matched to the findings of HRM over time. Isobaric contours in HRM were related to the specific structural regions on the pharynx and UES. Sensors were located on the same line of the soft palate (seventh), epiglottis (12th), and hypopharynx (13-17th) (Fig. 3A). About 1 second after swallowing, food bolus began to be pushed to the back of the mouth by the tongue, and the HRM catheter was displaced upward and backward (Fig. 3B). About 1.7 seconds after swallowing, a large amount of food remnants were noticed under the epiglottis on VFSE and increased pressure was detected by the sensor located on the mesopharynx. Next, increased pressure was detected by the 15th sensor located in the hypopharynx (Fig. 3C). Two seconds after swallowing, food remnants began to go through the hypopharynx and UES on VFSE, followed by hypopharyngeal muscular contraction on HRM (Fig. 3D). At 2.2 seconds after swallowing, a small amount of barium staining was noticed on the hypopharynx, and UES contraction was found on HRM (Fig. 3E). Three seconds after swallowing, barium staining on the hypopharynx was nearly gone on VFSE, and UES contraction almost stopped and returned to the basal state of UES on HRM (Fig. 3F).

\section{Effect of Food Viscosity on the Manometric Results During Pharyngeal Phase of Swallowing}

Table lists the mean $\pm \mathrm{SD}$ and median with IQR values for mesopharyngeal CI, hypopharyngeal CI, relaxation duration of UES, nadir pressure and median intrabolus pressure at UES of 3 swallow types. Manometric parameters were different for mesopharyngeal CI, hypopharyngeal CI and the median intrabolus pressure at UES after administration of $5 \mathrm{~mL}$ of water, barium and yogurt. Mesopharyngeal CIs for barium and yogurt swallows were significantly lower than those for water swallows $(P=$ 0.029 and $P=0.012$, respectively, Fig. 4). Also, hypopharyngeal CIs for water swallows were significantly lower than those for barium swallows ( $P=0.004$, Fig. 5). The median intrabolus pressure at UES for barium swallows was significantly higher than 
A

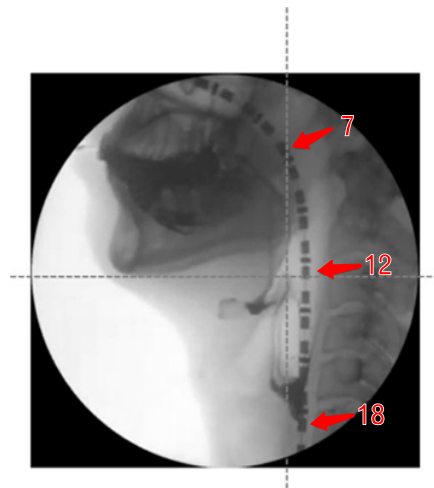

C

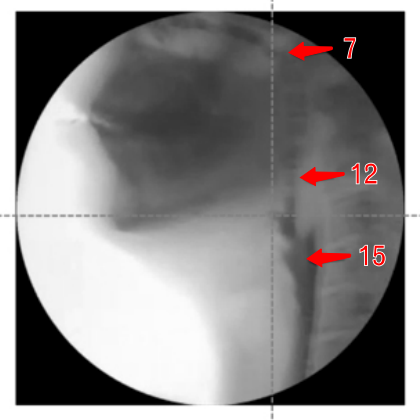

E

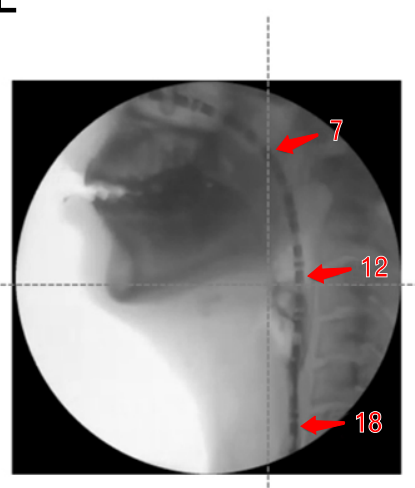

B

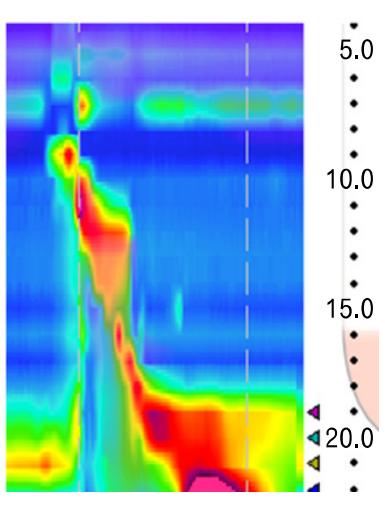

D
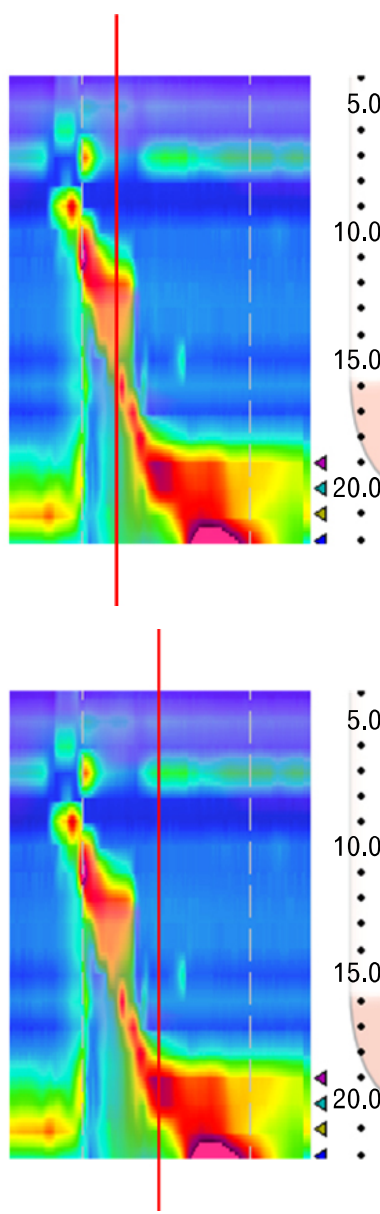

4

F
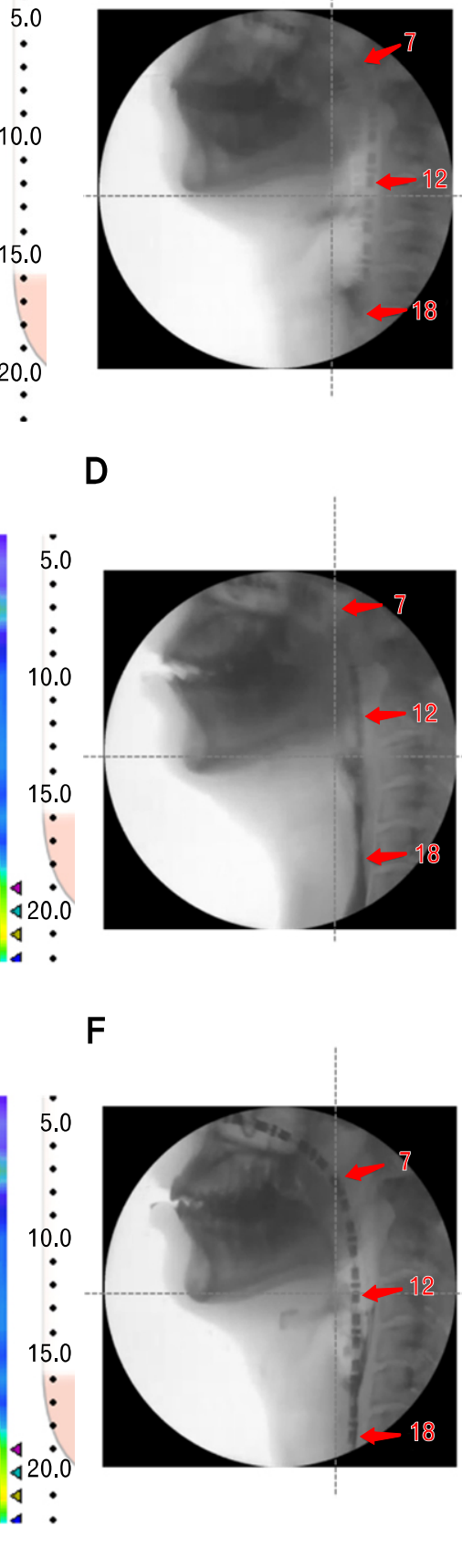
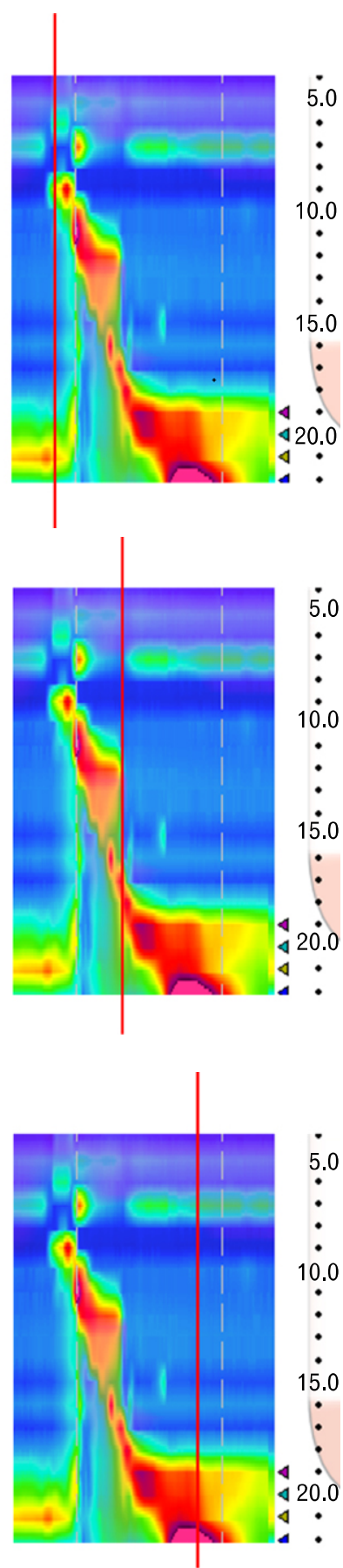

Figure 3. Simultaneous evaluation of videofluoroscopic swallow evaluation (VFSE) and high-resolution manometry (HRM). (A) Seventh, 12th and 13-17th sensors were located on the same line of the soft palate, epiglottis and hypopharynx, respectively (B) About 1 second after swallowing, the food bolus began to be pushed to the back of the mouth by the tongue, and the HRM catheter was displaced upward and backward (C) About 1.7 seconds after swallowing, a large amount of food remnants was noticed under the epiglottis on VFSE, and increased pressure was detected by the sensor located on the mesopharynx. Next, increased pressure was detected by the 15th sensor located in the hypopharynx (D) Two seconds after swallowing, food remnants began to go through the hypopharynx and UES on VFSE and hypopharyngeal muscular contraction started on HRM (E) At 2.2 seconds after swallowing, a small amount of barium staining was noticed on the hypopharynx and UES contraction was found on HRM (F) 3 seconds after swallowing, barium staining on the hypopharynx was nearly gone on VFSE, and UES contraction almost stopped on HRM. 

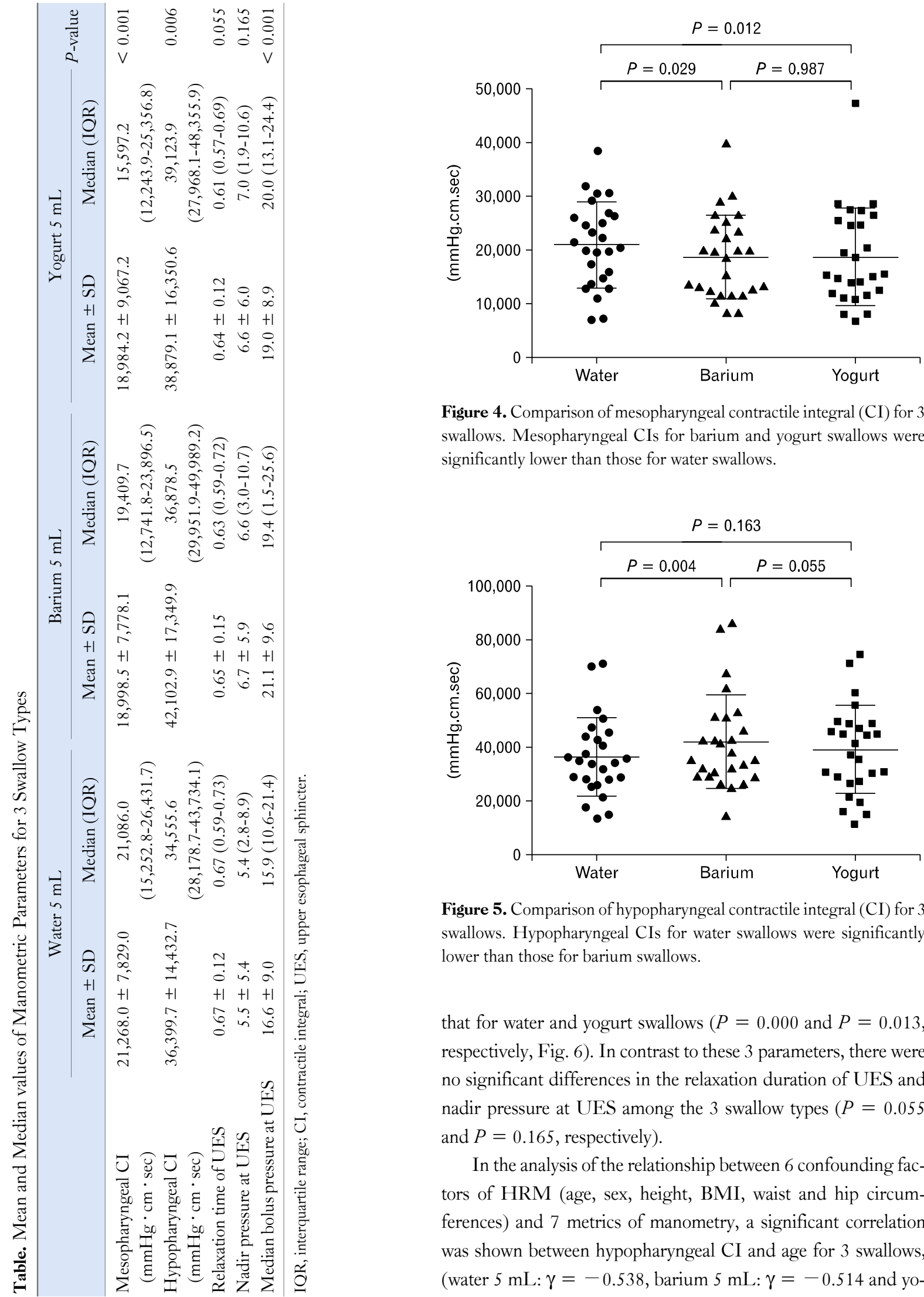

Figure 4. Comparison of mesopharyngeal contractile integral (CI) for 3 swallows. Mesopharyngeal CIs for barium and yogurt swallows were significantly lower than those for water swallows.

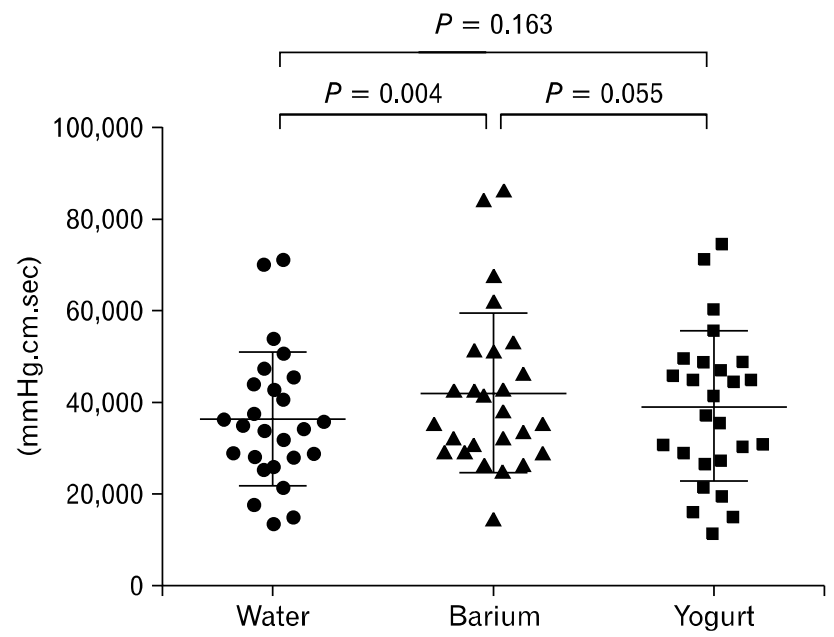

Figure 5. Comparison of hypopharyngeal contractile integral (CI) for 3 swallows. Hypopharyngeal CIs for water swallows were significantly lower than those for barium swallows.

that for water and yogurt swallows $(P=0.000$ and $P=0.013$, respectively, Fig. 6). In contrast to these 3 parameters, there were no significant differences in the relaxation duration of UES and nadir pressure at UES among the 3 swallow types $(P=0.055$ and $P=0.165$, respectively).

In the analysis of the relationship between 6 confounding factors of HRM (age, sex, height, BMI, waist and hip circumferences) and 7 metrics of manometry, a significant correlation was shown between hypopharyngeal $\mathrm{CI}$ and age for 3 swallows, (water $5 \mathrm{~mL}: \gamma=-0.538$, barium $5 \mathrm{~mL}: \gamma=-0.514$ and yo- 
gurt $5 \mathrm{~mL}: \gamma=-0.657$; all $P<0.01$, respectively; Fig. 7A), and between median intrabolus pressure at UES and age for 3 swallows, (water $5 \mathrm{~mL}: \gamma=-0.494$, barium $5 \mathrm{~mL}: \gamma=-0.559$ and

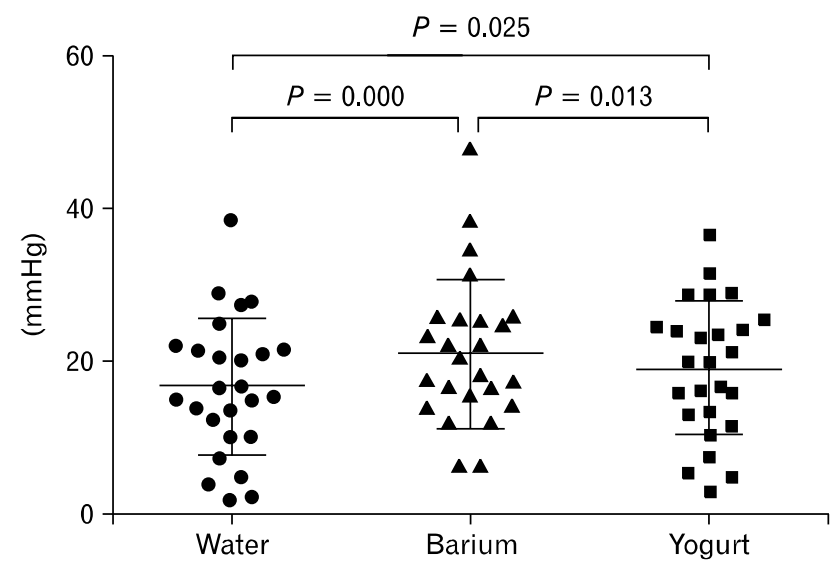

Figure 6. Comparison of median intrabolus pressure at upper esophageal sphincter (UES) for 3 swallows. Median intrabolus pressure at UES for barium swallows was significantly higher than that for water and yogurt swallows.
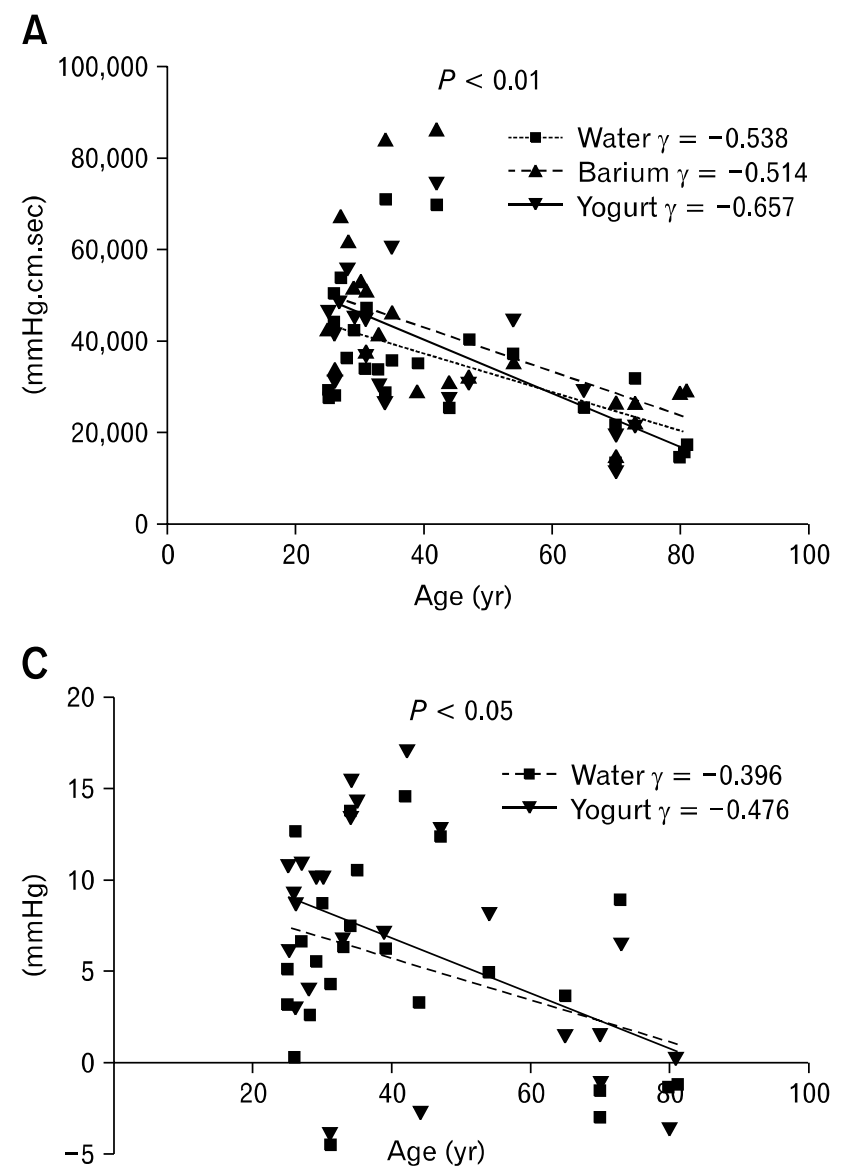

yogurt $5 \mathrm{~mL}: \gamma=-0.617 ; P=0.010, P=0.003$ and $P=$ 0.001 , respectively; Fig. 7B). Furthermore, nadir pressure at UES was significantly related to age for water and yogurt swallows (water $5 \mathrm{~mL}: \boldsymbol{\gamma}=-0.396$ and yogurt $5 \mathrm{~mL}: \boldsymbol{\gamma}=-0.476$; all $P<0.05$, respectively; Fig. $7 \mathrm{C}$ ). However, there was no significant relationship between age and basal UES pressure $(P>$ $0.05)$. No gender difference existed in the metrics for the 3 swallow types $(P>0.05)$.

\section{Discussion}

In the current study, we attempted to show the relationship between the results of HRM and VFSE by performing 2 tests simultaneously, and compared seven metrics of HRM among 3 swallow types: water, barium and yogurt (all $5 \mathrm{~mL}$ ). Differences were found in the values of mesophayngeal CI, hypopharyngeal $\mathrm{CI}$ and the median intrabolus pressure at UES. Furthermore, hypopharyngeal $\mathrm{CI}$ and median intrabolus pressure at UES were significantly related to age for all 3 swallows. A significant negative correlation was also noted between nadir pressure at UES

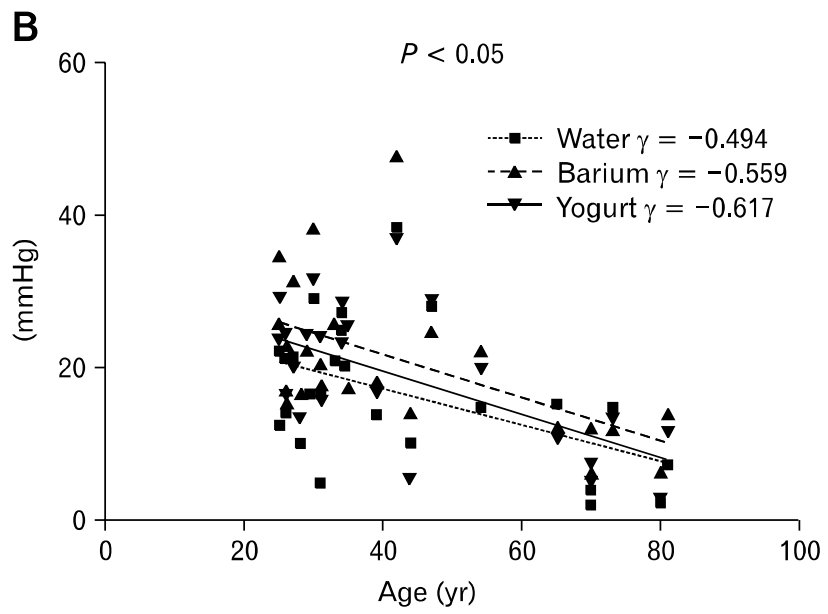

Figure 7. Correlation between metrics for 3 swallows and age. (A) Correlation was significant between hypopharyngeal contractile integral (CI) and age for 3 swallows, (all $P<0.01$, respectively) and (B) between median intrabolus pressure at UES and age for 3 swallows, $(P=0.010, P=0.003$ and $P=0.001$, respectively) $(\mathrm{C})$ Nadir pressure at upper esophageal sphincter was significantly related to age for water and yogurt swallows (all $P<0.05$, respectively). 
and age for water and yogurt swallows.

Most of our results were consistent with the results of earlier pharyngeal manometry studies. Values of UES relaxation interval, nadir pressure and intrabolus pressure of a previous report were quite similar to ours, and the finding that intrabolus pressure at UES was significantly increased with increased viscosity was also consistent with our results. ${ }^{18}$ In regard to UES relaxation interval, although some researchers have reported an increase in UES relaxation time with increasing viscosity, ${ }^{5}$ others have described no increase in UES relaxation. ${ }^{18-20}$ Similarly, no increase in nadir pressure at UES with increasing viscosity in the present study also coincides with previous observations. ${ }^{5,18}$ However, our findings were not always consistent with previous findings. Concerning pharyngeal contractility, greater mesopharyngeal CI for water swallowing was noted than that for yogurt or barium swallowing. This finding was not in agreement with previous reports that showed altered peak pharyngeal pressure in relation to volume, but not consistency, ${ }^{18}$ and no significant effects on viscosity and volume in peak pressures for the upper pharyngeal location. ${ }^{5}$ It is likely that the longer duration of mesopharyngeal contraction for water swallow than barium or yogurt swallow was responsible for this difference. Similarly, our finding that hypopharyngeal $\mathrm{CI}$, nadir pressure and median intrabolus pressure at UES were significantly decreased with age was not consistent with earlier studies that reported older adults had markedly higher nadir pressure at UES and pharyngeal contraction than younger adults. ${ }^{5,15}$ The reason for this is not clear; however, it might be due to the differences in demographic factors between populations studied such as age, obesity and racial background. ${ }^{21}$

Interestingly, the median intrabolus pressure for barium swallow was significantly greater than that for water and yogurt. Certainly, the finding that the median bolus pressure for barium and yogurt swallow was greater that for water swallow was consistent with previous findings. However, barium is less viscous compared to yogurt, and the median bolus pressure for barium should be lower than that for yogurt swallow. One possible explanation for this difference could be the fact that pressure was increased as the weight of the fluid increased. ${ }^{22}$ In the present study, the weight of barium $5 \mathrm{~mL}(128 \mathrm{~g})$ was almost twice as that of yogurt $(62 \mathrm{~g})$. Also, increased hypopharyngeal CI for barium swallow could be another explanation for this discrepancy.

Until recently, researchers have investigated pharyngeal contractility by estimating the peak pharyngeal pressure at 2 anatomical locations; upper and lower pharynx, or velo, meso-, or hypopharynx..$^{5,12,23}$ However, peak pharyngeal pressure only shows the highest pressure for a short time and it is difficult to say that it represents the contractility of the whole pharynx. CI was recently developed as a way of quantifying the overall vigor of contraction, and it is known to be sensitive in delineating abnormalities involving sustained or hypertensive esophageal contractions. ${ }^{13}$ In this study, we tried to make an adequate tool for the evaluation of pharyngeal and UES contractility based on the simultaneous examination of VFSE and HRM. CI was used for the quantification of contractility instead of peak pressure, and pharyngeal contraction segments on the HRM were divided into 2 structural lesions: meso- and hypopharynx based on the consuming time, feasibility and reproducibility.

According to the simultaneous examination of VFSE and HRM, it takes only $0.5-0.8$ seconds for food to pass through the mesopharynx and about 1 second to go through the hypopharynx. A significantly short time was required to complete swallowing and this made it difficult to divide the pharynx into more functionally distinct regions. Therefore, the pharynx was simply divided into 2 anatomical locations: the mesopharynx and the hypopharynx. Moreover, an accurate separation of the meso- and hypopharyngeal area on HRM was essential for the proper calculation of CI. With a reference value of $70 \mathrm{mmHg}$, the meso- and hypopharynx could be demarcated without difficulty. However, the hypopharynx and UES could not be separated functionally, since their contraction occurred successively in a very short period of time. As a result, CI of the hypopharynx was calculated along with that of UES. In addition, the contraction time for CI of hypopharynx and UES was determined as 2 seconds, since about 2 seconds were required for the complete evacuation of remnant food on VFSE (Fig. 3F). The muscle contraction time after the closure of UES was consistent with a previous report of a pharyngeal transit time of $1.01 \pm 0.24$ seconds for a $3 \mathrm{~mL}$ water swallow. ${ }^{24}$

In this study, UES relaxation parameters were measured using an established method. ${ }^{10}$ Median intrabolus pressure and nadir relaxation pressure could be calculated as previously reported. However, when relaxation interval was estimated, a few problems developed. First, the point of inflexion (defined as the point at which the slope of the curve changed from positive to negative) could change according to the usage of different equations (i.e., quadratic or cubic equation). Second, objective application of 3 line segments to the curve was not always possible. Third, the measuring method was too complex to apply to the HRM results of patients with dysphagia. Generally, HRM findings of patients with dysphagia were more complicated than those of normal 
subjects. Therefore, the old definition of UES relaxation interval was employed in this work.

A limitation may definitely exist in this method, including failure to draw a distinction between intrabolus pressure and muscular contraction pressure within the closed sphincter. In order to overcome this pitfall, the MATLAB program capable of showing isobaric contour with the line of half basal UES pressure and isoimpedance contour within the UES was developed. Figure 2B represents the isobaric and isoimpedance contour during the UES relaxation interval. As the isoimpedance line gets closer to the starting point of UES contraction, the intervals between lines become narrower and impedance increases. When relaxation comes to an end, low impedance lines change into lines with higher impedance, and the isoimpedance line changes direction toward the right side of the image, not downward (orange arrow). Although this method is a little subjective, it could be helpful to distinguish intrabolus pressure and the muscular contraction pressure of UES.

In the beginning of this study, a reference value for CI of mesopharynx was determined as a lower normal average pressure of pharynx. According to the descriptive statistics for the amplitude range during swallowing, pressure values tend to rise from the front to the back of the mouth. ${ }^{11}$ At the back of the mouth, the lower average pressure amplitude for water was about 40-50 $\mathrm{mmHg}$, and $40 \mathrm{mmHg}$ was selected as a reference value. However, with $40 \mathrm{mmHg}$, several problems occurred including difficulty in delineating bolus pressure and pressure by pharyngeal muscular contraction, and increased manometric artifacts resulting from external compression. Therefore, the upper average pressure amplitude, $70 \mathrm{mmHg}$, was chosen as a new reference value for mesopharyngeal CI. However, no significant differences were detected between mesopharyngeal CIs with the reference values $40 \mathrm{mmHg}$ and $70 \mathrm{mmHg}(P>0.05)$.

Similarly, there were several reasons why the reference value for hypopharyngeal CI was determined as $70 \mathrm{mmHg}$. First, researchers have reported that average resting UES pressure was $50-70 \mathrm{mmHg} .{ }^{12,16,25}$ Median resting UES pressure in our study was also very similar to that value; $78.8 \mathrm{mmHg}$ (range, 68.8-102.3). Second, if the reference value is set too low, it would be very difficult to distinguish muscular contraction by UES and upper esophageal sphincter. Lastly, $70 \mathrm{mmHg}$ was again used as the reference value for mesopharyngeal CI. Therefore, using the same criteria for hypopharyngeal and Hypopharyngeal CI with mesopharyngeal CI may be easier to remember and more convenient for use in clinical practice.
In conclusion, simultaneous examination of HRM and VFSE was helpful in understanding the swallowing mechanism. Manometric measurements of the pharynx and UES varied with respect to viscosity. Moreover, age could be a confounding variable in the interpretation of pharyngeal manometry. Therefore, future studies should aim to verify HRM with this analysis method to further understand the mechanism of dysphagia after considering the aforementioned confounding factors.

\section{References}

1. Dodds WJ, Stewart ET, Logemann JA. Physiology and radiology of the normal oral and pharyngeal phases of swallowing. Am J Roentgenol 1990;154:953-963.

2. Rubesin SE. Oral and pharyngeal dysphagia. Gastroenterol Clin North Am 1995;24:331-352.

3. Stoeckli SJ, Huisman TA, Seifert B, Martin-Harris BJ. Interrater reliability of videofluoroscopic swallow evaluation. Dysphagia 2003; 18:53-57.

4. Kelly AM, Drinnan MJ, Leslie P. Assessing penetration and aspiration: how do videofluoroscopy and fiberoptic endoscopic evaluation of swallowing compare? Laryngoscope 2007;117:1723-1727.

5. Butler SG, Stuart A, Castell D, Russell GB, Koch K, Kemp S. Effects of Age, Gender, Bolus Condition, Viscosity, and Volume on Pharyngeal and Upper Esophageal Sphincter Pressure and Temporal Measurements During Swallowing. J Speech Lang Hear Res 2009;52:240-253.

6. Kahrilas PJ, Dent J, Dodds WJ, Hogan WJ, Arndorfer RC. A method for continuous monitoring of upper esophageal sphincter pressure. Dig Dis Sci 1987;32:121-128.

7. Kahrilas PJ, Dodds WJ, Dent J, Logemann JA, Shaker R. Upper esophageal sphincter function during deglutition. Gastroenterology 1988;95:52-62.

8. Castell J, Castell D. Modern solid state computerized manometry of the pharyngoesophageal segment. Dysphagia 1993;8:270-275.

9. Pal A, Williams RB, Cook IJ, Brasseur JG. Intrabolus pressure gradient identifies pathological constriction in the upper esophageal sphincter during flow. Am J Physiol Gastrointest Liver Physiol 2003;285:G1037-G1048.

10. Ghosh SK, Pandolfino JE, Zhang Q, Jarosz A, Kahrilas PJ. Deglutitive upper esophageal sphincter relaxation: a study of 75 volunteer subjects using solid-state high-resolution manometry. Am J Physiol Gastrointest Liver Physiol 2006;291:G525-G531.

11. Steele CM, Bailey GL, Molfenter SM. Tongue pressure modulation during swallowing: water versus nectar-thick liquids. J Speech Lang Hear Res 2010;53:273-283.

12. Takasaki K, Umeki $\mathrm{H}$, Enatsu $\mathrm{K}$, et al. Investigation of pharyngeal swallowing function using high-resolution manometry. Laryngoscope 2008;118:1729-1732.

13. Ghosh SK, Pandolfino JE, Zhang Q, Jarosz A, Shah N, Kahrilas PJ. Quantifying esophageal peristalsis with high-resolution manometry: a study of 75 asymptomatic volunteers. Am J Physiol Gastrointest Liver Physiol 2006;290:G988-G997.

14. Kern M, Bardan E, Arndorfer R, Hofmann C, Ren J, Shaker R. 
Comparison of upper esophageal sphincter opening in healthy asymptomatic young and elderly volunteers. Ann Otol Rhinol Laryngol 1999;108:982-989.

15. van Herwaarden MA, Katz PO, Gideon RM, et al. Are manometric parameters of the upper esophageal sphincter and pharynx affected by age and gender? Dysphagia 2003;18:211-217.

16. Kwiatek M, Mirza F, Kahrilas P, Pandolfino J. Hyperdynamic upper esophageal sphincter pressure: a manometric observation in patients reporting globus sensation. Am J Gastroenterol 2009;104:289298.

17. Omari TI, Miki K, Davidson G, et al. Characterisation of relaxation of the lower oesophageal sphincter in healthy premature infants. Gut 1997;40:370-375.

18. Omari TI, Dejaeger E, Tack J, Van Beckevoort D, Rommel N. Effect of bolus volume and viscosity on pharyngeal automated impedance manometry variables derived for broad Dysphagia patients. Dysphagia 2013;28:146-152.

19. Dantas RO, Dodds WJ. Influence of the viscosity of the swallowed food bolus on the motility of the pharynx. Arq Gastroenterol 1990;27:164-168.

20. Dantas RO, Kern MK, Massey BT, et al. Effect of swallowed bolus variables on oral and pharyngeal phases of swallowing. Am J Physiol Gastrointest Liver Physiol 1990;258(5 Pt 1):G675-G681.

21. Sweis R, Anggiansah A, Wong T, Kaufman E, Obrecht S, Fox M. Normative values and inter-observer agreement for liquid and solid bolus swallows in upright and supine positions as assessed by esophageal high-resolution manometry. Neurogastroenterol Motil 2011;23: 509-e198.

22. Ambaum MHP. General relationships between pressure, weight and mass of a hydrostatic fluid. Philos Trans R Soc Lond A 2008;464: 943-950.

23. Takasaki K, Umeki H, Hara M, Kumagami H, Takahashi H. Influence of Effortful Swallow on Pharyngeal Pressure: Evaluation Using a High-Resolution Manometry. Otolaryngol Head Neck Surg 2011;144:16-20.

24. Kendall KA, McKenzie S, Leonard RJ, Goncalves MI, Walker A. Timing of events in normal swallowing: a videofluoroscopic study. Dysphagia 2000;15:74-83.

25. Babaei A, Bhargava V, Mittal RK. Upper esophageal sphincter during transient lower esophageal sphincter relaxation: effects of reflux content and posture. Am J Physiol Gastrointest Liver Physiol 2010; 298:G601-G607. 